\title{
The superSTEM: An Aberration Corrected Analytical Microscopy Facility
}

\author{
A. Bleloch, L.M. Brown, R. Brydson*, A. Craven ${ }^{* *}$, P. Goodhew ${ }^{* * *}$ and C. Keily ${ }^{* * *}$. \\ University of Cambridge, Cavendish Laboratory, Madingley Rd., Cambridge CB3 OHE, UK \\ *Materials, University of Leeds, Leeds, LS2 9JT, UK \\ **Physics and Astronomy, The Kelvin Building, University of Glasgow, Glasgow, G12 8QQ, UK \\ ****aterials Science and Engineering, University of Liverpool, Liverpool L69 3GH, UK
}

Following the initial demonstration of aberration correction in scanning transmission electron microscopy (STEM) by Krivanek[1] and the 'synchrotron in a microscope' paper by Brown[2], the United Kingdom electron microscopy community organized and supported a bid to set up a national facility to exploit the technology of aberration correction for the benefit of the national and international community.

Hitherto, a great deal of our understanding of the optics of the electron microscope has been dominated by the spherical aberration of the objective lens, for example choosing the optimal aperture and interpreting images in terms of the contrast transfer function. The advent of practicable spherical aberration correction in two laboratories in the last decade[1,3] has changed the way in which we approach many of these tasks. The relative merits and areas of application of conventional transmission electron microscopy (CTEM) and scanning transmission electron microscopy (STEM) are a part of this new debate. Building an aberration corrector into a STEM makes particular use of the strengths of that instrument; namely, an optimal configuration for high angle annular dark field imaging (HAADF) coupled with electron energy loss spectroscopy (EELS).

In figure 1 the probe size is plotted against the beam convergence angle. Each line on the plot represents a constraint due either to diffraction or one or other aberration. This plot gives one perspective on the 'new territory' that has become available as a result of the technology of aberration correction. It is not strictly correct to simply add the different contributions in quadrature to produce an overall constraint, but more detailed simulations have shown that, to within a factor of two, this is a justifiable approach and is very useful in developing a new set of heuristics[4]. The lesson from the plot is that there is not only the optimal resolution apex of the 'new territory' but a whole area of different convergence angle vs. probe-size and probe current trade-offs that are now accessible.

One significant omission from figure 1 is the role of instabilities in limiting the performance of the instrument. Instabilities caused by electrical interference, vibration (and sound), and thermal variations are, to a good approximation, independent random variables, and so adding them in quadrature is justifiable. As a consequence, once the dominant resolution limitation is removed (usually spherical aberration), several others become evident as limiting. In order to be able to use the new instruments to their designed performance routinely and to be able to cope with future innovations, the instruments should therefore be housed in a building that itself performs to the highest level. The superSTEM laboratory building has been specified and designed with these objectives in mind and is currently under construction.

An integral part of the superSTEM facility is the provision of remote access to the instruments. We have performed some demonstration experiments from which we have learned that the instrument 
will be operated most effectively as a three-way conference between the remote user, the local operator and the instrument, rather than having autonomous remote operation of all parameters.

Below, figure 2a shows an image of gold particles taken with the first superSTEM corrector that will be installed in March 2002[5]. Lattice fringes are evident in most of the orientations in these multiply twinned particles. Analysis of the diffractogram shows transfer of spatial information down to $0.115 \mathrm{~nm}$ in this annular dark field (ADF) image. The enhanced contrast version of the image in figure $2 \mathrm{~b}$ shows rafts of gold atoms joining the particles as well as a few scattered single gold atoms.

\section{References}

1. L. M. Brown, (1997), Inst. Phys. Conf. Ser. No. 153, 17-22

2. O. L. Krivanek, N. Delby and A. R. Lupini, (1999), Ultramicroscopy, 78, 1-11

3. M. Haider et al., (1998), Nature, 392, 768

4. A. R. Lupini, Ph.D. thesis (2000), Cambridge University

5. Image courtesy of Nion Co., Kirkland, WA taken during testing.

6. We would like to thank the EPSRC, CLRC Daresbury, EMAG (IOP), and the RMS.

Figure 1.Probe size vs. convergence angle. The shaded area is now accessible.

Figure 2. An ADF image of gold particles acquired with $100 \mathrm{keV}$ electrons. Figure $2 b$ is $2 a$ contrast enhanced. Features ascribed to single atoms are visible (see arrow).
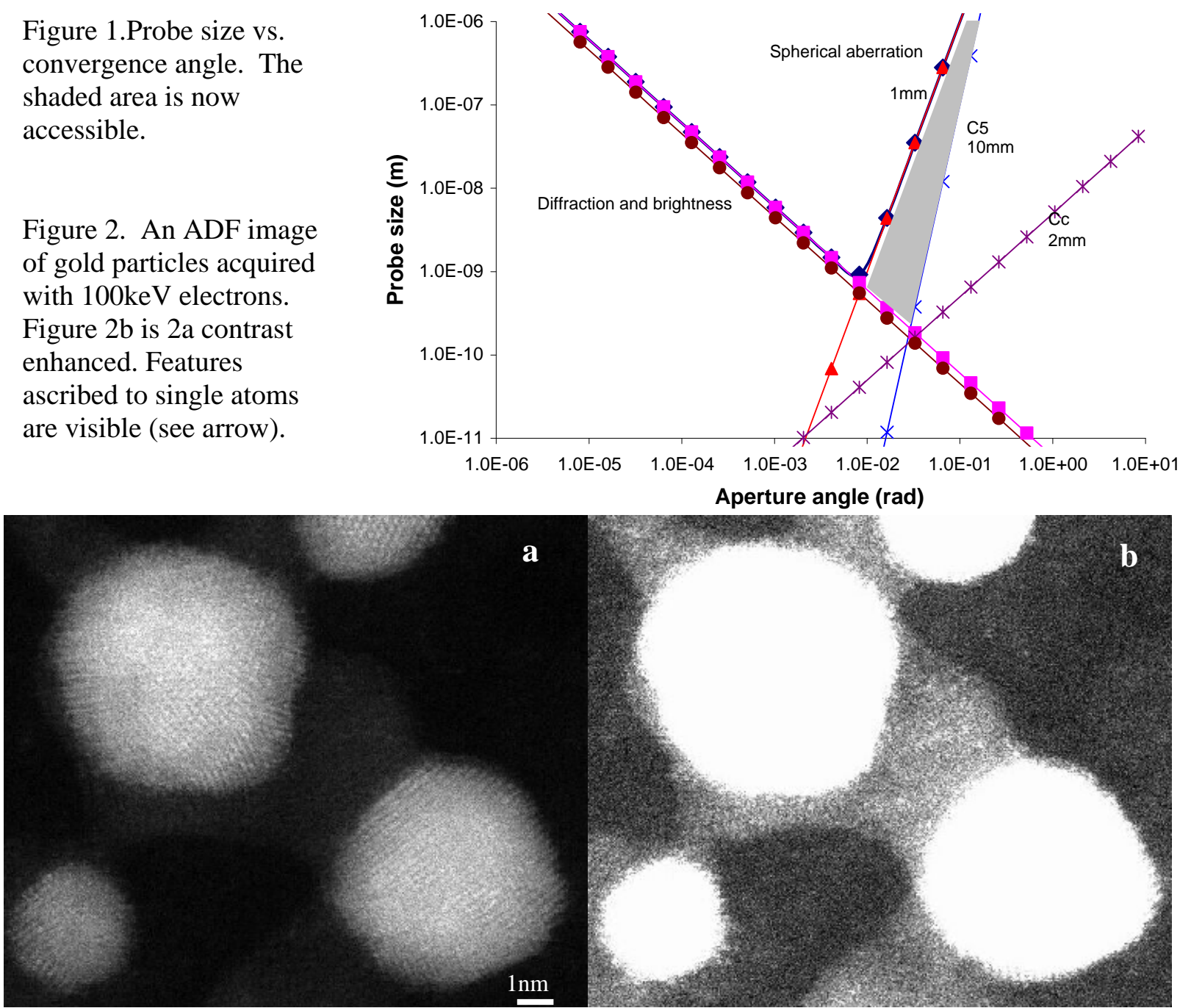